\title{
Online Linear Optimization with Inventory Management Constraints
}

\author{
Lin Yang* \\ The Chinese University of Hong \\ Kong, China \\ Hong Kong \\ linyang@cs.umass.edu \\ Adam Wierman \\ California Institute of Technology, \\ USA \\ Pasadena, CA \\ adamw@caltech.edu
}

\author{
Mohammad H. Hajiesmaili* \\ University of Massachusetts Amherst, \\ USA \\ Amherst, MA \\ hajiesmaili@cs.umass.edu \\ Enrique Mallada \\ Johns Hopkins University, USA \\ Baltimore, MD \\ mallada@jhu.edu
}

\author{
Ramesh Sitaraman \\ University of Massachusetts Amherst, \\ USA \\ Amherst, MA \\ ramesh@cs.umass.edu \\ Wing S. Wong \\ The Chinese University of Hong \\ Kong, China \\ Hong Kong \\ wswong@ie.cuhk.edu.hk
}

\begin{abstract}
This paper considers the problem of online linear optimization with inventory management constraints. Specifically, we consider an online scenario where a decision maker needs to satisfy her timevarying demand for some units of an asset, either from a market with a time-varying price or from her own inventory. In each time slot, the decision maker is presented a (linear) price and must immediately decide the amount to purchase for covering the demand and/or for storing in the inventory for future use. The inventory has a limited capacity and can be used to buy and store assets at low price and cover the demand when the price is high. The ultimate goal of the decision maker is to cover the demand at each time slot while minimizing the cost of buying assets from the market. We propose ARP, an online algorithm for linear programming with inventory constraints, and ARPRate, an extended version that handles rate constraints to/from the inventory. Both ARP and ARPRate achieve optimal competitive ratios, meaning that no other online algorithm can achieve a better theoretical guarantee. To illustrate the results, we use the proposed algorithms in a case study focused on energy procurement and storage management strategies for data centers.
\end{abstract}

\section{CCS CONCEPTS}

- Theory of computation $\rightarrow$ Online algorithms; Linear programming; • Hardware $\rightarrow$ Energy generation and storage; Power estimation and optimization.

\section{KEYWORDS}

Online linear optimization; inventory management; competitive online algorithms; energy procurement; data center

${ }^{*}$ Both authors contributed equally to this research.

Permission to make digital or hard copies of part or all of this work for personal or classroom use is granted without fee provided that copies are not made or distributed for profit or commercial advantage and that copies bear this notice and the full citation on the first page. Copyrights for third-party components of this work must be honored.

For all other uses, contact the owner/author(s).

SIGMETRICS '20 Abstracts, fune 8-12, 2020, Boston, MA, USA

(C) 2020 Copyright held by the owner/author(s)

ACM ISBN 978-1-4503-7985-4/20/06.

https://doi.org/10.1145/3393691.3394207

\section{ACM Reference Format:}

Lin Yang, Mohammad H. Hajiesmaili, Ramesh Sitaraman, Adam Wierman, Enrique Mallada, and Wing S. Wong. 2020. Online Linear Optimization with Inventory Management Constraints. In ACM SIGMETRICS / International Conference on Measurement and Modeling of Computer Systems (SIGMETRICS '20 Abstracts), fune 8-12, 2020, Boston, MA, USA. ACM, New York, NY, USA, 1 page. https://doi.org/10.1145/3393691.3394207

The full version of this abstract is available in [1].

\section{ACKNOWLEDGMENTS}

This work was funded by the National Science Foundation through the CNS-1908298, CNS-1763617, AitF-1637598, CNS-1518941, CPS1544771, EPCN-1711188, CAREER-1752362, AMPS-1736448, TRIPODS1934979 grants, and ARO: W911NF-17-1-0092, DoE: ENERGISE-DEEE0008006 grants, and a Google Faculty Research Award. Lin Yang wants to acknowledge the support from Schneider Electric, Lenovo Group (China) Limited and the Hong Kong Innovation and Technology Fund (ITS/066/17FP) under the HKUST-MIT Research Alliance Consortium.

\section{REFERENCES}

[1] L. Yang, M. H. Hajiesmaili, R. Sitaraman, A. Wierman, E. Mallada, and W. S. Wong. Onlinelinearoptimizationwithinventorymanagementconstraints. Proc. ACM Meas. Anal. Comput. Syst., 4(1):Article16, 2020. 